\section{d'Interactions Non-Linéaires*}

\author{
P. Chen, G. Horton-Smith \\ Stanford Linear Accelerator Center, \\ Stanford University, Stanford, CA 94309 \\ T. Ohgaki \\ Department of Physics, Hiroshima University, \\ Higashi-Hiroshima 724 \\ A. W. Weidemann ${ }^{\dagger}$ \\ Department of Physics and Astronomy, \\ University of Tennessee, Knoxville, TN 37996-1200 \\ K. Yokoya \\ KEK - National Laboratory for High-Energy Physics, \\ 1-1 Oho, Tsukuba-shi, Ibaraki-ken 305
}

\section{Abstract}

We present our plans for a Monte-Carlo code simulating all possible combinations of (electromagnetic) interactions between colliding electron, positron, and both high-energy and laser photon beams, based on the ABEL code for beam-beam interaction. The implementation. and first results for the laser- $e^{-}$interaction are described.

"Work supported by the Department of Energy contract DE-AC-76SF00515 (SLAC) and DE-FG05-91ER40627 (Tennessee).

†Mailing Address: SLAC, MS 94, P.O.B.4349, Stanford, CA 94309.

Contributed to the Workshop on Gamma-Gamma Colliders, Berkeley, CA, March 28-31, 1994
1. Introduction

Detailed knowledge of the beam-beam interaction in cc lisions of electrons, positrons and photons at high-energy colliders is important for the estimation of expected backgrounds and as an area of investigation in its own right.

The necessity to focus the beams to a small spot at the interaction point to achieve an acceptable luminosity deforms the beam much more than at current electron-positron storage rings, and requires a deeper understanding of all aspects of the interactions of the two beams.

For $e^{+} e^{-}$colliders, the ABEL code (Analysis of Beam-beam Effects in Linear colliders)[1] allows a simulation of the beam-beam interaction including disruption and beamstrahlung effects.

As electron-positron pair creation in the collisions of the intensive beams of future colliders poses potential background problems for high-energy physics [2], the ABEL code had been subsequently modified to include incoherent pair creation in the equivalent photon approximation ("ABELMOD")[3].

In the $\gamma \gamma$ colliders considered at this workshop, the high-energy photon beam is made by the Compton scattering of a laser beam off a high-energy electron beam. For lasers of sufficiently high intensity, non-linear Compton effects can occur, where several laser photons are absorbed in exchange for a high-energy final state photon. Such nonlinear effects are currently investigated in an experiment [4] at the Final Focus Test Beam at the Stanford Linear Accelerator Center.

Therefore, an extension of ABEL to include laser- $e^{-}$beam scattering, both linear and non-linear, is of great topical interest now already; and an extension including all possible classical and quantum electrodynamic interactions between electrons, positrons, and photons is clearly needed for $\gamma \gamma$ collider studies. Indeed, a $\gamma \gamma$ collider with the spent electrons (used to make the high-energy photons) not swept out of the interaction region has the most complicated conceivable beam-beam interaction, which possibly could be simulated by such an extension of ABEL.

In Section 2. below we list the particles and interactions an all-encompassing Monte Carlo code should be able to handle. We chose to call this code 'CAIN' (as shown in the title) to indicate both its origin in the ABEL code and the importance of non-linear effects. In Section 3, we describe our first efforts to include Compton scattering in an extension of ABEL and show some simulation results for Compton scattering.

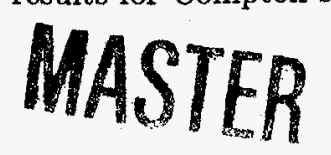

1

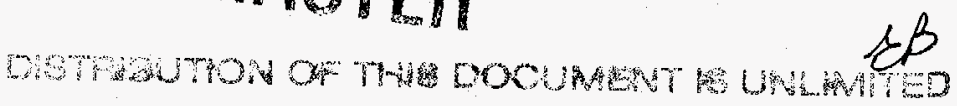




\section{Plans for CAIN}

The purpose of CAIN is to describe all possible interactions, due to both classical and quantum electrodynamics, between high-energy beams of electrons, positrons and photons, and external electromagnetic fields.

Indeed all possible combinations of particles and fields do occur in the various schemes proposed for $\gamma \gamma$ collider regions.

If the electron beams (used to make the high-energy photon beam) are deflected by a bend magnet, the residual fields of the deflected beams are still felt at the $\gamma \gamma$ interaction point [5][2]. In another scheme, where the electrons comove with the high-energy photon beam, and are disrupted by a previously introduced offset ("heads-up" collisions) [6], all four beams are present at the $\gamma \gamma$ interaction point. This configuration also occurs when the spent electron beam is over-focussed by a plasma lens [7], although the electron beams here are diluted.

Finally, it would be desirable if the code would also be able to deal with the interactions of the various beams with a plasma lens [8].

To achieve all these goals, the following particles and fields are involved:

1. Particles: $e^{+}, e^{-}, \gamma$

2. External fields (static fields, laser fields, and plasmas);

3. Internal fields (the Coulomb field created by the $e^{+}$and $e^{-}$).

We assume that the particles $\left(e^{+}, e^{-}, \gamma\right)$ have high energies $\left(\gg m_{e} c^{2}\right)$, and are approximately left- or right-moving along some axis (s-axis), as appropriate for linear-collider studies. The laser beam may have an arbitrary direction with respect to the particle beam.

The particles, either left- or right-moving, are represented by macroparticles with three space and three momentum coordinates, and a spin vector. Their initial distribution is specified by appropriate beam and beam optics parameters. For high-energy beams these are the beam energy, bunch population, beam rms size, beam emittance, the Twiss parameters, transverse offset, crossing angle, degree of crab crossing etc.; for laser beams, the wavelength, peak intensity, pulse length, transverse size, angular divergence, orientation of the polarization vector, and the Stokes parameters.
The following interactions of these particles and fields are to be included in the CAIN code:

1. Interaction of $e^{ \pm}$with external static E,B fields, and collective fields in plasmas;

2. $e^{ \pm}+$laser $\rightarrow e^{ \pm}+\gamma$;

a routine for Compton scattering already exists; it takes the local laser intensity and polarization effects into account; nonlinear QED processes, such as a nonlinear Compton scattering (Volkov [9] and postVolkov [10] formulae), as well as $e^{ \pm}+$laser $\rightarrow e^{ \pm} e^{+} e^{-}$will be included soon.

3. $\gamma+$ laser $\rightarrow e^{+} e^{-}$

a routine for Breit-Wheeler scattering invoking local laser intensity and polarization effects already exists; again, nonlinear effects are to be included soon.

4. Interaction of $e^{ \pm}$and the internal fields:

$e^{ \pm}$tracked using equations of motion;

classical precession of spin;

beamstrahlung;

These already exist in ABEL. At each time step $\Delta t$, the s-coordinate of each macroparticle changes by $\pm c \Delta t$. Because of the assumptions, only charged particles with almost the same s-coordinate feel their mutual Coulomb forces; thus at each time step, the transverse distribution of the particles in each longitudinal slice is used to solve a two-dimensional Poisson equation from which the Coulomb force is calculated.

5. Interaction of photons and the internal/external fields: coherent pair production;

6. Incoherent particle-particle interactions:

Bremsstrahlung: $e e \rightarrow e e \gamma$;

Breit-Wheeler: $\gamma \gamma \rightarrow e^{+} e^{-}$

Bethe-Heitler: $e \gamma \rightarrow e e^{+} e^{-}$; 


\section{DISCLAIMER}

This report was prepared as an account of work sponsored by an agency of the United States Government. Neither the United States Government nor any agency thereof, nor any of their employees, make any warranty, express or implied, or assumes any legal liability or responsibility for the accuracy, completeness, or usefulness of any information, apparatus, product, or process disclosed, or represents that its use would not infringe privately owned rights. Reference herein to any specific commercial product, process, or service by trade name, trademark, manufacturer, or otherwise does not necessarily constitute or imply its endorsement, recommendation, or favoring by the United States Government or any agency thereof. The views and opinions of authors expressed herein do not necessarily state or reflect those of the United States Government or any agency thereof. 


\section{DISCLAIMER}

Portions of this document may be illegible in electronic image products. Images are produced from the best available original document. 
Landau-Lifshitz: $e e \rightarrow e e e^{+} e^{-}$;

In ABELMOD, these interactions are already fully implemented, whereas in the ABEL version on which CAIN is based certain higher-order ef-

fects are currently neglected, but will also be included soon.

\section{Details of Laser-Electron Interaction}

Due to the interest by a current experiment [4] we already began implementing the laser-electron interaction. Here one has to consider that the angular divergence of the laser beam is important. Even at the focal point, where there is no correlation of the mean angle with position and the envelope of the laser pulse is parallel, the laser photons can come from a range of directions within the aperture of the focussing optics. The laser intensity depends on position and time; this is important especially for non-linear effects.

A routine has already been written which calculates the local direction of laser propagation, the local average intensity, and the local angular divergence (in the transverse coordinates $x, y$ ). The routine allows several choices for the laser beam profile:

1. transverse gaussian, longitudinal uniform;

2. square and divergenceless (which is clearly unphysical, as violating Huygen's law, but useful for some checks);

3. transverse gaussian, longitudinal uniform as in 1., but with smearing of photon momenta according to the calculated local angular divergence.

The local quantities of the laser field are then used as inputs to a Compton event generator or a Breit-Wheeler event generator; the latter two routines also take polarization effects into account.

In the figures below we show some first results obtained for laser-Compton scattering. The parameters were chosen to be close to those considered in [4]: The laser beam crosses the electron beam with a mean angle of $17^{\circ}$. The laser beam has a $\mathrm{f} / \mathrm{d}$ ratio of 4 ; the smallest spot size has a diameter of $7 \mu \mathrm{m}$. The full length of the laser pulse is $600 \mu \mathrm{m}(2 p s)$, the total laser power is 1 Joule; the laser wavelength $526 \mathrm{~nm}$. The electron beam energy is $46.6 \mathrm{GeV}$; its rms sizes are $\sigma_{r} \approx 14 \mu m$ and $\sigma_{z} \approx 500 \mu m$. To show the effects of the tight laser focus, the electron beam was taken to be emittanceless and perfectly parallel, that is without any angular divergence.

For Figures 1 the trajectories of the scattered electrons (or photons) were projected back to their intercept with the plane at $z=0$ and their positions there given as a scatterplot. The hyperbolic shape of the focus of the laser can be made out.

Figures 2 and 3 show the shape of the energy spectrum of scattered electrons and photons, respectively, near the kinematic edge for first-order Compton scattering.

We observe that this edge is washed out due to the distribution of crossing angles which the electrons encounter as they pass through the tightly focussed laser beam.

\section{Conclusions}

In this note, we presented our plans for CAIN and a first result for laser Compton scattering. Clearly, we are now only at the beginning of this project and much more work needs to be done.

\section{Acknowledgments}

We thank Dr. M. Ronan of LBL for discussions at the inception of this project, and for providing a modularized form of the ABEL program. We thank the organizers of the Gamma-Gamma Collider Workshop for having organized a very successful and inspiring meeting.

\section{References}

[1] K. Yokoya, KEK-Report 85-9, October 1985; also Nucl.Instr.Meth. A251 (1986) 1.

[2] P. Chen and V. I. Telnov, Phys. Rev. Lett. 63 (1989) 1796.

[3] T. Tauchi, K. Yokoya, P. Chen, Part. Acc. 41 (1993) 29.

[4] J.G. Heinrich, C. Lu, K.T. McDonald, C. Bamber, A.C. Melissinos, D. Meyerhofer, Y. Semertzidis, Pisin Chen, J.E. Spencer, R.B. Palmer, 
"Proposal for a Study of QED at Critical Field Strengths in Intense Laser-High-Energy Electron Collisions," SLAC-Proposal-E-144, October 1991.

[5] V. I. Telnov, Nucl.Instr.Meth. A294 (1990) 72.

[6] V. E. Balakin, these Proceedings.

[7] S. Rajagopalan, D.B. Cline, P. Chen, "Application of a Plasma Lens to $e^{-} \gamma$ Colliders," these Proceedings.

[8] Plasma Lens Collaboration (W. Barletta et al.), "Proposal for Plasma Lens Experiments at the Final Focus Test Beam," SLAC-Proposal-E150, April 1993.

[9] V. B. Berestetskii, E. M. Lifshitz, L. P. Pitaevskii, Quantum Electrodynamics, Section 101 (Pergamon Press 1982).

[10] S. Ben-Menachem and P. Chen, Proceedings of the Fifth Int. Workshop on Next-Generation Linear Colliders, SLAC-436, October 1993, p. 426.

\section{Figure Captions}

Fig. 1. Scatterplot of origins of scattered $e^{-}$or $\gamma$ in the transverse $(x, y)$ plane.

Fig. 2. Energy spectrum of scattered $e^{-}$near Compton edge. (Ordinate is energy/incident $e^{-}$energy) The dots show the simulation result, the dashed line indicates the kinematic edge.

Fig. 3. Energy spectrum of scattered $\gamma$ near Compton edge. (Ordinate is energy/incident $e^{-}$energy) The dots show the simulation result, the dashed line indicates the kinematic edge. 


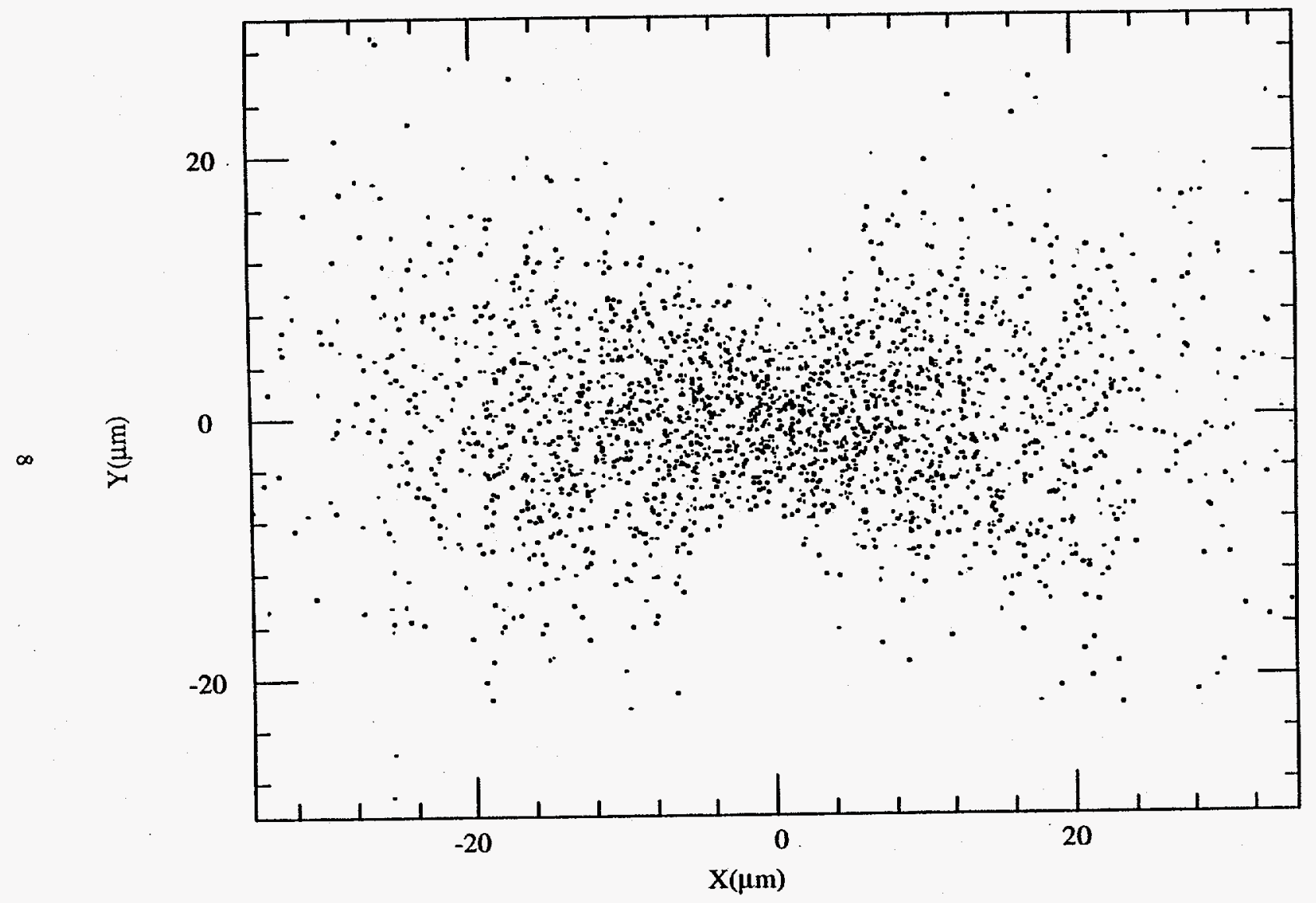

Fig. 1.

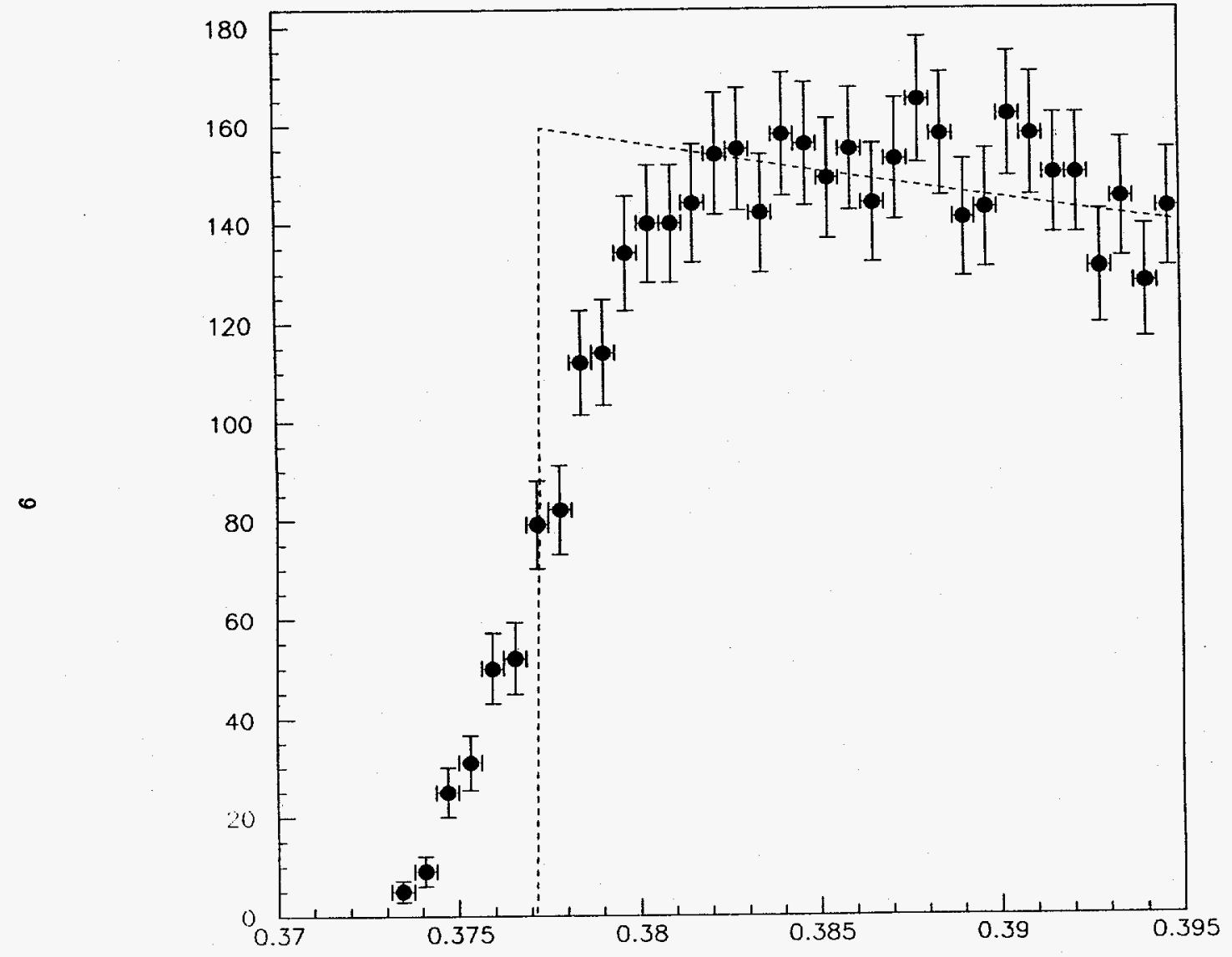

Fig. 2. 


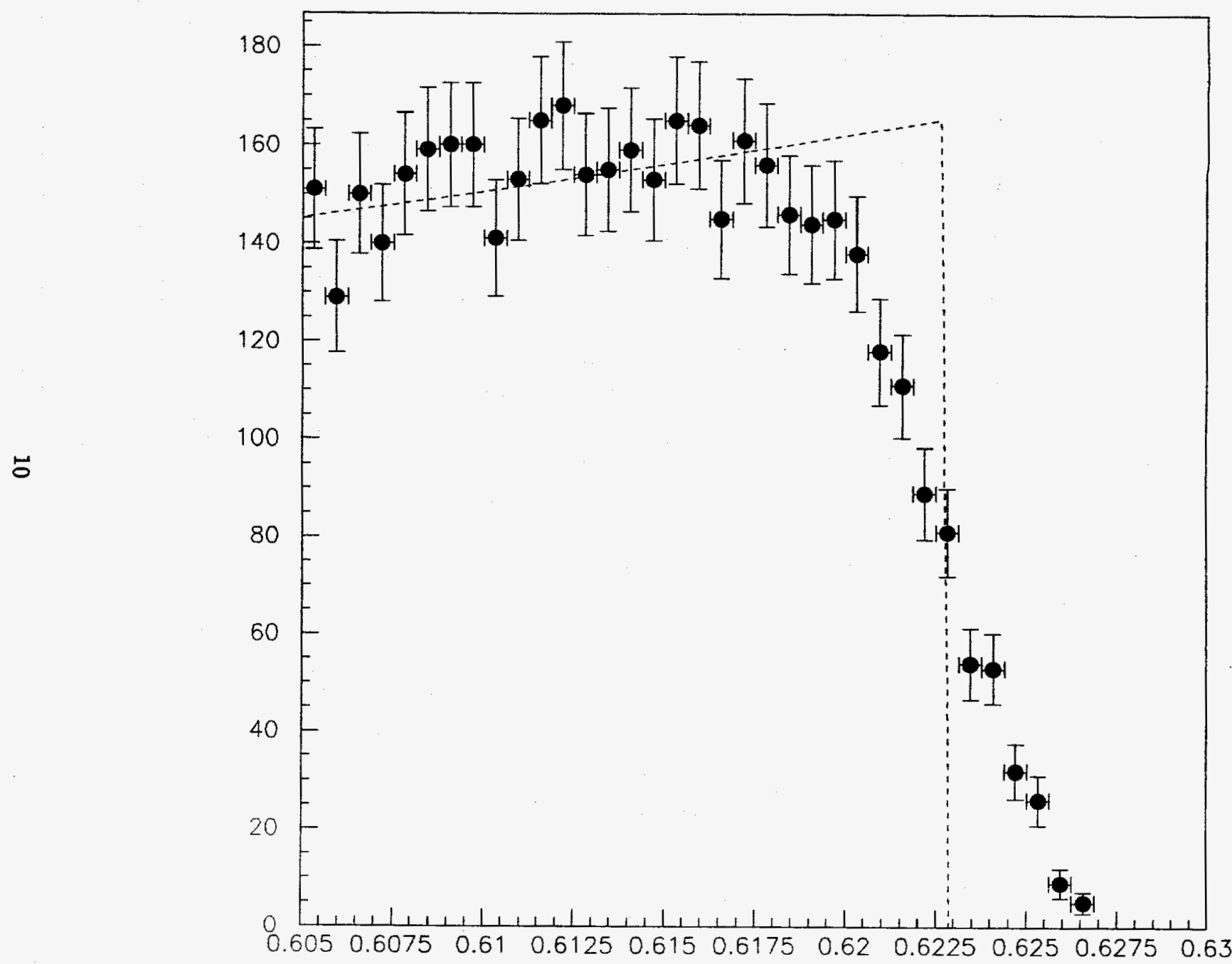

Fig. 3. 